\title{
Surgery for pheochromocytoma: A 20-year experience of a single institution
}

\author{
Chrysanthi Aggeli, ${ }^{1}$ Alexander M. Nixon, ${ }^{1}$ Christos Parianos, ${ }^{1}$ \\ Georgios Vletsis, ${ }^{1}$ Labrini Papanastasiou, ${ }^{2}$ Athina Markou, ${ }^{2}$ \\ Theodora Kounadi, ${ }^{2}$ Georgrios Piaditis, ${ }^{2}$ Georgios N. Zografos ${ }^{1}$
}

${ }^{1}$ Third Department of Surgery, Athens General Hospital "G. Gennimatas", Greece; 'Department of Endocrinology and Diabetes Center, Athens General Hospital "G. Gennimatas", Greece

\begin{abstract}
OBJECTIVE: Resection of pheochromocytomas is a challenging procedure due to hemodynamic lability. Our aim was to evaluate surgical outcomes in 67 patients with pheochromocytoma and to validate the role of laparoscopic surgery in the treatment of these tumors. DESIGN: This study is a retrospective review. A total of 68 procedures for pheochromocytoma were performed between June 1997 and February 2017. All patients were investigated and operated on using an established departmental protocol. Relevant data were retrieved from the hospital records of 533 patients who underwent 541 adrenalectomies for benign and malignant adrenal tumors in the same period. RESULTS: Sixty-nine tumors were removed from 67 patients. One patient with/MEN2A underwent bilateral resection of pheochromocytomas in two stages. Tumor size in laparoscopic procedures ranged from $1.2 \mathrm{~cm}$ to $11.0 \mathrm{~cm}$ (mean $5.87 \mathrm{~cm})$. Thirty-seven patients had benign disease, 31 potentially malignant (based on PASS) and 1 malignant with metastasis. Eight were in the context of a familial syndrome. Forty-nine patients underwent laparoscopic adrenalectomy, 8 patients had open approach from the start for recurrent pheochromocytoma or large benign tumor, 1 patient had open approach due to inoperable malignant pheochromocytoma and 10 patients had conversions from laparoscopic to open procedure. Nine patients received sodium nitroprusside intraoperatively to treat hypertension. One patient developed pulmonary embolism and succumbed 1 month later. There were no recurrences of the benign or potentially malignant tumors during the follow-up period. CONCLUSIONS: Laparoscopic resection of pheochromocytomas, despite its increased level of difficulty compared to that of other adrenal tumors, is a safe and effective procedure.
\end{abstract}

Key words: Endocrine surgery, Laparoscopic adrenalectomy, Pheochromocytoma

Address for correspondence:

George N. Zografos, MD, Consultant Surgeon, 10 K. Ourani Str., 15237 Athens, Greece; Tel.: +30944918944,

Fax: +302107706915, E-mail: gnzografos@yahoo.com

Received: 14-03-2017, Accepted: 11-11-2017

\section{INTRODUCTION}

Pheochromocytoma (PHEO) is a tumor derived from chromaffin cells of the sympathetic nervous system and its clinical symptoms are associated with 
excessive production and release of catecholamines which can cause arterial hypertension and symptoms attributed to paroxysmal stimulation of the adrenergic system. ${ }^{1,2}$

Since the first report on laparoscopic adrenalectomy by Gagner in 1992, this approach has increasingly become the gold standard for excision of benign functioning and non-functioning tumors of the adrenal gland. ${ }^{3}$ Laparoscopic adrenalectomy compared to traditional open resection has proven equally safe and efficient, offering a number of significant advantages such as improved access to the surgical area, less blood loss, decreased requirement for postoperative analgesia, shorter hospital stay and earlier return to normal diet and activities. ${ }^{4-6}$

On the other hand, in large PHEOs technical difficulties increase due to possible hemodynamic instability, tumor vascularity and malignant potential. Given the technical challenges for resection of large PHEOs, there were hesitations about using a laparoscopic approach for these tumors during the first decade of laparoscopic surgery. Meanwhile however, improvement in imaging modalities, better pharmacological preparation, advances in anaesthesia and laparoscopic surgery as well as evolving technology rendered laparoscopic surgery for PHEO safe and efficient. $^{7}$

\section{MATERIALS AND METHODS}

A total of 68 procedures for PHEO were performed between June 1997 and February 2017. Four abdominal paragangliomas operated on during the same period are not included in this series. The preoperative diagnosis, operative details, complications, length of hospital stay, morbidity and follow-up were retrieved from the hospital records of 533 patients who underwent 541 adrenalectomies for benign and malignant adrenal tumors in the same period. Since 2008, data have been retrieved from our Department database including prospective patient records.

One patient with MEN2A syndrome underwent bilateral tumor resection with left cortex-preserving adrenalectomy.

Preoperative localization was established in all patients by computerized tomography (CT) or mag- netic resonance imaging (MRI), while iodine-123 metaiodobenzylguanidine (MIBG) scan was reserved for ambiguous cases where paraganglioma or metastatic disease was suspected. All patients were investigated according to an established protocol drawn up by the Department of Endocrinology and Diabetes Center at our Hospital.

Endocrinological evaluation and complete adrenal dynamic testing were performed to determine whether the tumor was functional or not. All patients underwent basal adrenal hormonal investigation including serum cortisol, adrenocorticotropin (ACTH), dehydroepiandrosteronesulphate, renin and aldosterone levels, and the aldosterone/renin ratio. The evaluation of cortisol hypersecretion included 24-h urinary free cortisol and measurement of serum cortisol and ACTH levels after an overnight dexamethasone suppression test $(1 \mathrm{mg}$ of dexamethasone administrated at midnight, before the morning blood sample). The evaluation of PHEOs included the measurement of urinary fractioned metanephrines (normetanephrines and metanephrines) as per local availability of diagnostic tests. Measurements of plasma free or urinary fractionated metanephrines are superior to other tests of catecholamine excess for the diagnosis of pheochromocytomas ${ }^{8}$ and, until there are data directly comparing plasma and urinary measurements, there is no recommendation that one test is superior to the other.

In all patients, $\alpha$-adrenergic blockade with a titration dose of phenoxybenzamine (starting dose 10mg - mean dose $100 \mathrm{mg}$ - max dose $200 \mathrm{mg}$ ) was administered for 7-14 days prior to surgery to achieve a blood pressure of approximately $120 / 80 \mathrm{mmHg}$. High sodium diet and intravenous fluids were administered after the second or third day of the initiation of medical treatment in order to achieve volume expansion and counteract the orthostasis associated with $\alpha$-adrenergic blockade. In cases of tachyarrhythmias, a $\beta$-adrenoceptor blocker was added after the $\alpha$-adrenergic blockade.

All patients had blood pressure less than $140 / 80 \mathrm{~mm}$ $\mathrm{Hg}$, to omit orthostatic hypotension not exceeding $80 / 45 \mathrm{mmHg}$, no more than one ventricular extrasystole every 5 minutes and ECG without nonspecific ST segment elevations or depression as well as $\mathrm{T}$ wave inversions, thus ensuring adequate preoperative preparation. 


\section{SURGICAL TECHNIQUE}

All the operations, open or laparoscopic, were performed with the patient in the lateral decubitus position. Therefore, there was no need to change the position of the patient in the event of a conversion to an open operation. A transperitoneal lateral approach was used to perform laparoscopic adrenalectomy.

High technology instruments and equipment are mandatory and available in the operating theater. We use a monitor tower and gas insufflator set at an intra-abdominal pressure of $14 \mathrm{mmHg}$ and a $30^{\circ} 10 \mathrm{~mm}$ laparoscope. We prefer to create pneumoperitoneum with the Hasson technique to avoid any relevant morbidity. The adrenal gland is never grasped, especially in cases of PHEOs, to avoid hemodynamic instability, troublesome bleeding or tumor disruption. We ligate small vessels with clips or the harmonic scissors/ scalpel. We use additional ports in obese patients or when there is a special need. Occasionally, in large tumors we use the hand-assisted technique as the last effort to avoid conversion. The specimen is placed in a special bag and extracted through minimal extension of the initial incision.

We consider early ligation of the adrenal vein to reduce the risk of hemodynamic instability. However, delayed adrenal vein ligation, following tumor mobilization, is equally safe provided that efficient pharmacological preparation is completed.

\section{RESULTS}

Sixty-nine tumors were removed from 67 patients (34 men, 33 women), with a mean age of 53.0 years (range 16-76). The mean hospital stay was 2.1 days (range 2-3 days) for the laparoscopic procedures.

The diagnosis included 37 benign PHEOs, 31 potentially malignant (based on PASS $\geq 4$ ) and 1 malignant with metastasis. Malignancy was defined solely based on the presence of metastasis and not on a history of local recurrence, in accordance with the World Health Organization's classification of tumors. ${ }^{9}$ One patient had von Recklinghausen disease and 2 patients had Von Hippel-Lindau syndrome. One patient with MEN2A underwent bilateral resection of PHEOs in two stages. Tumor size in laparoscopic procedures ranged from $1.2 \mathrm{~cm}$ to $11.0 \mathrm{~cm}$ (mean 5.87 $\mathrm{cm})$. Operative time ranged from 55 to 210 minutes (mean 90 minutes).

Forty-eight patients underwent laparoscopic adrenalectomy, 1 patient underwent hand-assisted laparoscopic adrenalectomy, 8 patients had an open approach from the beginning due to either recurrent or large tumors (mean tumor size $=6.1 \mathrm{~cm}$ ), 1 patient had an open biopsy due to inoperable malignant PHEO and there were 10 conversions from laparoscopic to open adrenalectomy. Six conversions were performed for hemostasis and 4 due to periadrenal tissue invasion. The relative majority of conversions ( 5 out of 8 laparoscopies, $62.5 \%$ ) occurred in the first 5 years of this series. In the last 15 years the conversion rate dropped significantly (5 out of 51 laparoscopies, $9.8 \%$ ). These conversions were undertaken in order to achieve safe oncological margins and only one for hemorrhage control.

Blood transfusion was needed only in 2 patients, 1 with resection of recurrent malignancy and 1 due to preoperative anemia and minimal controlled bleeding.

Nine patients (5 laparoscopic adrenalectomies and 4 open) received sodium nitroprusside because of intraoperative hypertensive crisis (defined as a systolic blood pressure over $220 \mathrm{mmHg}$ sustained over 10 minutes). All significant episodes were noted during manipulation of the tumor and before ligation of the adrenal vein. Shorter episodes of hypertension that did not require pharmacological treatment with vasodilators were not included in these results. Sodium nitroprusside has a rapid onset of action but also end/ termination of effect and should only be administered under close monitoring. All 9 patients had been treated with the same protocol of phenoxybenzamine titration as described above.

Transient episodes of arrhythmia or tachycardia (duration $<30$ s) were not documented as part of the study protocol and no sustained episodes (duration $>30$ s) of abnormal cardiac rhythm or frequency were observed. An interesting exception was a female patient with a negative preoperative work-up for PHEO and therefore no blockade, who presented hemodynamic instability during the operation and finally proved to have a potentially malignant $\mathrm{PHEO}$ with a PASS $=6$ (PHEO of the Adrenal gland Scaled Score). 
Postoperatively, there were no life-threatening complications, except for one patient who after right laparoscopic adrenalectomy developed pulmonary embolism. The patient was hospitalized in the intensive care unit and succumbed a month later.

All patients showed remarkable improvements in hypertension and reversal of the characteristic symptoms of constant adrenoreceptor stimulation by catecholamines.

No clinically significant episodes of postoperative hypoglycemia were documented.

At a mean follow-up interval of 152 months after laparoscopic adrenalectomy (range 2 months -19.5 years), resolution of hormonal activity and no evidence of tumor recurrence were documented. The patient with the recurrent malignant PHEO survived for 2 years after the last operation. Thirty-one tumors with potentially malignant features on pathology as assessed by PASS were identified, but follow-up has not demonstrated thus far any evidence of recurrence or metastasis.

\section{DISCUSSION}

We reviewed our experience with operative treatment of pheochromocytoma to demonstrate the safety and efficacy of the laparoscopic approach and to present some noteworthy issues concerning the management of these patients. Before the advent of preoperative adrenergic blockade, morbidity and mortality associated with hemodynamic lability due to excessive catecholamine secretion were prohibitively high. Operative mortality in the pre-blockade era was reported at $13-45 \%$, primarily due to myocardial infarction and cerebrovascular accidents. ${ }^{15}$ Preoperative blockade has significantly reduced adverse outcomes but has not completely eliminated episodes of hemodynamic lability. ${ }^{16}$

Proper preparation of the patient before surgery is crucial. The preparation includes the administration of $\alpha$ - with or without $\beta$-blockers and intravenous expansion with crystalloids. In particular, nonselective $\alpha$-adrenergic blockade (phenoxybenzamine) and selective $\alpha 1$-adrenergic blockade (prazocin) are used for $\alpha$-blockade. ${ }^{17,18}$ In cases of co-existing tachycardia $\beta$-blockade is employed. Intravenous volume expan- sion with crystalloids $(2000 \mathrm{ml} /$ day starting on the day before surgery) is of critical importance. Continuous invasive monitoring and pharmacologic intervention by an experienced anesthetic team perioperatively are necessary to avoid substantial cardiovascular instability. ${ }^{19}$ When the main adrenal vein is ligated, antihypertensive drugs and $\beta$-blockers administration are stopped and hemodynamics are reassessed..$^{20,21}$

At our institution we avoid aggressive pharmacological vasodilation, especially before ligation of the adrenal vein, in order to avoid an abrupt drop in blood pressure. ${ }^{16}$ Transient spikes in blood pressure are usually managed by limiting tumor manipulation. Guiding this practice is the fact that tumor manipulation has been shown to be the most significant intraoperative stimulus for catecholamine release during both open and laparoscopic resections. ${ }^{27,30}$ Limiting tumor manipulation and applying pharmacological vasodilation when hypertension is persistent has resulted in no cardiovascular morbidity or mortality in our series. The sole exception was one patient who developed a severe pulmonary embolus and died after being hospitalized for a month. It should be noted that no intraoperative hemodynamic lability was observed. To our knowledge no other patient developed any form of clinically evident deep vein thrombosis. Interestingly, the incidence of clinically evident deep vein thrombosis has been reported to be as high as $7 \%$ in laparoscopic adrenalectomy. ${ }^{31}$

It has been suggested that the laparoscopic approach to PHEO may decrease the intraoperative release of catecholamines, compared with open surgery, thus reducing the risk of a hypertensive crisis. ${ }^{32}$ This may be a result of fewer operative manipulations, although it seems a somewhat hyperbolic suggestion. Laparoscopy offers improved visualization and faster access to the adrenal vein further reducing the risk of catecholamine release. Other studies suggest that intra-abdominal insufflation during laparoscopy may alone cause an increase in serum catecholamines..$^{28,33}$ This is probably the result of direct tumor compression or alteration in tumor perfusion. ${ }^{28}$

Early ligation of the adrenal vein has historically been suggested as a prophylactic factor minimizing hormonal secretion. ${ }^{34}$ By contrast, others endorse the safety of delayed adrenal vein ligation, challenging 
the importance of the long-standing tradition of early adrenal vein ligation for these patients. ${ }^{35,36}$ In our opinion, this technique may be even safer because the tumor is mobilized from the inferior vena cava to the right and the renal vein to the left and thus possible hemorrhage is better controlled by laparoscopy. Nonetheless, we always contemplate an initial effort to dissect the main adrenal vein at an early stage of the procedure but, if safety is jeopardized, we follow the delayed vein ligation technique. ${ }^{37}$

Tumor size is another consideration in laparoscopic adrenalectomy in general. In the case of PHEO, guidelines published by the Endocrine Society suggest open adrenalectomy as the treatment modality of choice in PHEOs larger than $6 \mathrm{~cm}$ due to increased concerns for obtaining safe oncological margins and the perceived increased risk of capsule rupture. ${ }^{38}$ A recent limited non-randomized controlled study of large pheochromocytomas $(>6 \mathrm{~cm})$ comparing laparascopic adrenalectomy and open adrenalectomy indicated that recurrence rates were not statistically different between the two groups, thus questioning current guidelines. ${ }^{39}$ However, sample sizes were relatively small and larger studies are required to fully validate laparoscopic adrenalectomy in large PHEOs. It should be highlighted that tumor size has not been conclusively proven to be a reliable predictor of recurrence or malignant potential, with different studies yielding opposing conclusions..$^{10,40}$ In our study the largest PHEO excised laparoscopically was $11.0 \mathrm{~cm}$ in diameter. We had 4 conversions to an open procedure because of periadrenal tumor invasion. During our follow-up there have been no instances of local recurrence. However, PHEO recurrence or metastasis can develop as long as 20 years after surgical excision and therefore all patients need to be monitored long-term.

Tumor size may also be a concern regarding conversion rates in laparoscopic surgery. However, the most common incidental cause of conversion in adrenalectomy in general is iatrogenic vascular injury. ${ }^{14,41,42}$ As previously mentioned, PHEOs are characterized by a rich network of vessels and thus carry a higher probability of intraoperative troublesome hemorrage (Figure 1). In our series we had 6 conversions to an open procedure for successful hemostasis in patients with troublesome but not massive bleeding. It seems that laparoscopic adrenalectomy of large PHEOs is safe as long as the surgeon has the appropriate experience. ${ }^{43}$ When using $4 \mathrm{~cm}$ as a cut-off value between small and large tumors there does not seem to be a significant difference in operative time and surgical outcomes. ${ }^{44}$ In light of this, laparoscopic adrenalectomies have been performed for non-PHEO tumors up to $15 \mathrm{~cm}$ in diameter without any significant morbidity, therefore PHEO size does not seem to preclude a laparoscopic approach. ${ }^{45-49}$

Suspected malignancy remains a controversial issue. ${ }^{50}$ Thus far, the only universally accepted criterion for malignancy in the case of PHEO is metastasis. ${ }^{9}$ Currently there is no definitive way to predict which tumors will progress to malignancy. If there are no metastases it is impossible to precisely evaluate the degree of malignancy based on biochemical and imaging tests alone. ${ }^{51}$

Even pathologic evaluation is non-definitive. The most popular scale employed for estimating the risk of malignancy is the PASS, which was developed by Thompson in 2002..$^{51,52}$ Thompson proposed that tumors with a PASS score $\geq 4$ should be considered biologically more aggressive. Nevertheless, it is has been demonstrated that this system cannot precisely

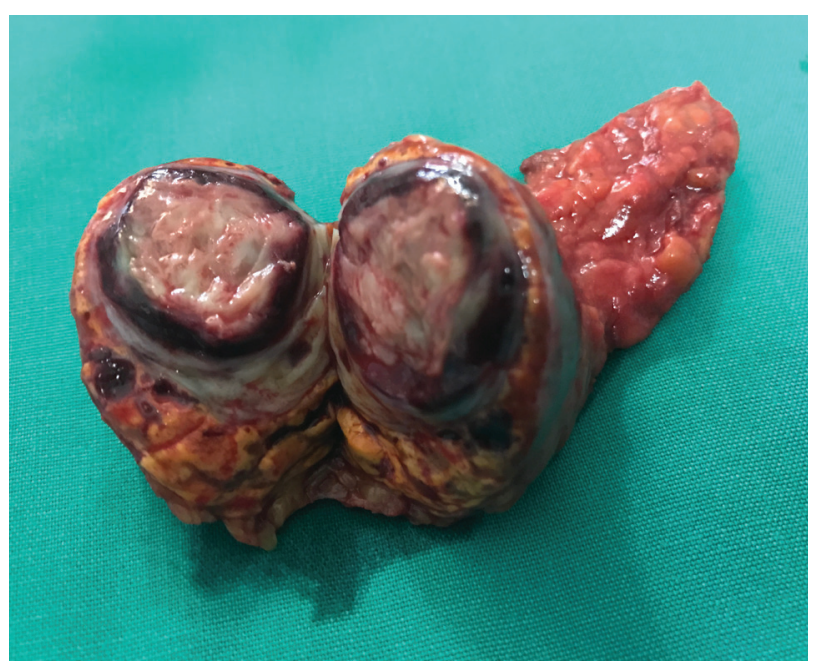

Figure 1. Benign adenoma of the right adrenal gland. Hemorrhagic infiltration of the adrenal cortex is evident. Preoperative imaging indicated a pheochromocytoma. 
diagnose malignancy or predict the postoperative course after adrenalectomy. ${ }^{51,53}$

PHEOs exhibit a modest response to radiotherapy and systemic therapy (e.g. MIGB) ${ }^{54}$ Surgical resection is currently the only therapeutic option. ${ }^{55}$ However, there are concerns about the ability of minimally invasive techniques to totally remove the malignant lesion and avoid capsular disruption of the tumor during dissection. Laparoscopic adrenalectomy for potentially malignant (or locally aggressive) PHEOs can be performed in appropriately selected cases, in experienced centers with oncologic outcomes that are equivalent to open approaches, while providing advantages in terms of patient morbidity. ${ }^{56}$ Proponents of minimally invasive techniques argue that in the hands of experienced surgeons, laparoscopy can be safely performed while preserving the principles of oncologic surgery, with results similar to those of open access. The basic principles during surgery are to avoid direct manipulation of or application of pressure to the tumor in order to avoid rupture of the tumor capsule. ${ }^{51,57}$ The adrenal gland is resected en bloc with the surrounding adipose tissue and it is always extracted after being placed in a specimen retrieval bag. ${ }^{51}$

PHEO has been proposed as an independent factor predicting open conversion. ${ }^{14}$ In our series our conversion rate reached $17.2 \%$, higher than that of the laparoscopic approach for other benign adrenal lesions $(8.06 \%) .{ }^{61}$ However, after the eighth laparoscopic attempt conversion rates dropped significantly to $9.8 \%$ (from $62.2 \%$ ), indicating that familiarity with the intricacies of this procedure have a profound effect on successful completion by laparoscopy and might indicate different learning curves compared to other adrenal tumors. When taking into account the 9.8\% conversation rate, no statistical significance is noted compared to other benign adrenal lesions. The early conversion rates may be partially explained by the low threshold to convert to an open procedure during the first attempts rather than struggle to complete a laparoscopic operation by risking disruption of the tumor capsule.

In conclusion, laparoscopic adrenal resection represents a safe and effective option for the management of PHEOs. Patient selection, close preoperative and intraoperative management and adequate postoperative follow-up are essential to prevent surgery-induced uncontrollable catecholamine secretion and cardiovascular instability. Laparoscopic resection of large PHEOs necessitates experience in open and advanced laparoscopic surgery. Potentially malignant tumors should not be accounted as an absolute contraindication for laparoscopic excision but oncological principles should be strictly followed. Special effort should be made not to damage the capsule of the tumor. Nonetheless, large potentially malignant or malignant tumors $>10 \mathrm{~cm}$, or those with possible organ infiltration must be approached with the open technique from the beginning.

\section{CONFLICT OF INTEREST}

The authors have no conflict of interest to declare.

\section{REFERENCES}

1. Pacak K, Eisenhofer G, Ahlman H, et al, 2007 Pheochromocytoma: recommendations for clinical practice from the First International Symposium, October 2005. Nat Clin Pract Endocrinol Metab 3: 92-102.

2. Kaltsas GA, Papadogias D, Grossman AB, 2004 The clinical presentation (symptoms and signs) of sporadic and familial chromaffin cell tumours (phaeochromocytomas and paragangliomas). Front Horm Res 31: 61-75.

3. Gagner M, Lacroix A, Bolte E, 1992 Laparoscopic adrenalectomy in Cushing's syndrome and pheochromocytoma. N Engl J Med 327: 1033.

4. Assalia A, Gagner M, 2004 Laparoscopic adrenalectomy. Br J Surg 91: 1259-1274.

5. Dudley NE, Harrison BJ, 1999 Comparison of open posterior versus transperitoneal laparoscopic adrenalectomy. Br J Surg 86: 656-660.

6. Wang HS, Li CC, Chou YH, Wang CJ, Wu WJ, Huang $\mathrm{CH}, 2009$ Comparison of laparoscopic adrenalectomy with open surgery for adrenal tumors. Kaohsiung J Med Sci 25: 438-444.

7. Brunaud L, Nguyen-Thi PL, Mirallie E, et al, 2015 Predictive factors for postoperative morbidity after laparoscopic adrenalectomy for pheochromocytoma: a multicenter retrospective analysis in 225 patients. Surg Endosc 30: 1051-1059.

8. Lenders WMJ, Duh Quan-Yang, Eisenhofer G, et al 2014 Pheochromocytoma and Paraganglioma:An Endocrine Society Clinical Practice Guideline J Clin Endocrinol Metab 99: 1915-1942.

9. DeLellis RA, 2004 International Agency for Research 
on Cancer, World Health Organization, International Academy of Pathology, Pathology and genetics of tumours of endocrine organs.

10. Amar L, Servais A, Gimenez-Roqueplo AP, Zinzindohoue F, Chatellier G, Plouin PF, 2005 Year of diagnosis, features at presentation, and risk of recurrence in patients with pheochromocytoma or secreting paraganglioma. J Clin Endocrinol Metab 90: 2110-2116.

11. Pillinger SH, Bambach CP, Sidhu S, 2002 Laparoscopic adrenalectomy: a 6-year experience of 59 cases. ANZ J Surg 72: 467-470.

12. Tiberio GA, Baiocchi GL, Arru L, et al, 2008 Prospective randomized comparison of laparoscopic versus open adrenalectomy for sporadic pheochromocytoma. Surg Endosc 2008: 1435-1439.

13. Lee J, El-Tamer M, Schifftner T, et al, 2008 Open and laparoscopic adrenalectomy: analysis of the National Surgical Quality Improvement Program. J Am Coll Surg 206: 953-961.

14. Shen ZJ, Chen SW, Wang S, et al, 2007 Predictive factors for open conversion of laparoscopic adrenalectomy: a 13-year review of 456 cases. J Endourol 21: 1333-1337.

15. Desmonts JM, Le Houelleur J, Remond P, Duvaldestin P, 1977 Anaesthetic management of patients with phaeochromocytoma. A review of 102 cases. Br J Anaesth 49: 991-998.

16. Ramakrishna H, 2015 Pheochromocytoma resection: Current concepts in anesthetic management. J Anaesthesiol Clin Pharmacol 31: 317-323.

17. Siddiqi HK, Yang HY, Laird AM, et al, 2012 Utility of oral nicardipine and magnesium sulfate infusion during preparation and resection of pheochromocytomas. Surgery 152: 1027-1036.

18. Combemale F, Carnaille B, Tavernier B, et al, 1998 Exclusive use of calcium channel blockers and cardioselective beta-blockers in the pre- and per-operative management of pheochromocytomas. 70 cases. Ann Chir 52: 341-345.

19. Kiernan CM, Du L, Chen X, et al, 2014 Predictors of hemodynamic instability during surgery for pheochromocytoma. Ann Surg Oncol 21: 3865-3871.

20. Natkaniec M, Pedziwiatr M, Wierdak M, et al, 2015 Laparoscopic adrenalectomy for pheochromocytoma is more difficult compared to other adrenal tumors. Wideochir Inne Tech Maloinwazyjne 10: 466-471.

21. Mann C, Millat B, Boccara G, Atger J, Colson P, 1996 Tolerance of laparoscopy for resection of phaeochromocytoma. Br J Anaesth 77: 795-797.

22. Gagner M, Breton G, Pharand D, Pomp A, 1996 Is laparoscopic adrenalectomy indicated for pheochromocytomas? Surgery 120: 1076-1080.

23. Janetschek G, Finkenstedt G, Gasser R, et al, 1998 Laparoscopic surgery for pheochromocytoma: adrenalectomy, partial resection, excision of paragangliomas. J Urol 160: 330-334.
24. Edwin B, Kazaryan AM, Mala T, Pfeffer PF, Tonnessen TI, Fosse E, 2001 Laparoscopic and open surgery for pheochromocytoma. BMC Surg 1: 2.

25. Inabnet WB, Pitre J, Bernard D, Chapuis Y, 2000 Comparison of the hemodynamic parameters of open and laparoscopic adrenalectomy for pheochromocytoma. World J Surg 24: 574-578.

26. Kocak S, Aydintug S, Canakci N, 2002 Alpha blockade in preoperative preparation of patients with pheochromocytomas. Int Surg 87: 191-194.

27. Kercher KW, Novitsky YW, Park A, Matthews BD, Litwin DE, Heniford BT, 2005 Laparoscopic curative resection of pheochromocytomas. Ann Surg 241: 919928.

28. Flavio Rocha M, Faramarzi-Roques R, Tauzin-Fin P, Vallee V, Leitao de Vasconcelos PR, Ballanger P, 2004 Laparoscopic surgery for pheochromocytoma. Eur Urol 45: 226-232.

29. Kinney MA, Warner ME, vanHeerden JA, et al, 2000 Perianesthetic risks and outcomes of pheochromocytoma and paraganglioma resection. Anesthesia and Analgesia 91: 1118-1123.

30. Lang B, Fu B, OuYang JZ, et al, 2008 Retrospective comparison of retroperitoneoscopic versus open adrenalectomy for pheochromocytoma. J Urol 179: 57-60.

31. Shen WT, Grogan R, Vriens M, Clark OH, Duh QY, 2010 One hundred two patients with pheochromocytoma treated at a single institution since the introduction of laparoscopic adrenalectomy. Arch Surg 145: 893-897.

32. Ramacciato G, Paolo M, Pietromaria A, et al, 2005 Ten years of laparoscopic adrenalectomy: lesson learned from 104 procedures. Am Surg 71: 321-325.

33. Joris JL, Hamoir EE, Hartstein GM, et al, 1999 Hemodynamic changes and catecholamine release during laparoscopic adrenalectomy for pheochromocytoma. Anesthesia and Analgesia 88: 16-21.

34. Janetschek G, Neumann HP, 2001 Laparoscopic surgery for pheochromocytoma. Urol Clin North Am 28: 97-105.

35. Zhang X, Lang B, Ouyang JZ, et al, 2007 Retroperitoneoscopic adrenalectomy without previous control of adrenal vein is feasible and safe for pheochromocytoma. Urology 69: 849-853.

36. Vassiliou MC, Laycock WS, 2009 Laparoscopic adrenalectomy for pheochromocytoma: take the vein last? Surg Endosc 23: 965-968.

37. Zografos GN, Farfaras AK, Kassi E, et al, 2011 Laparoscopic resection of pheochromocytomas with delayed vein ligation. Surg Laparosc Endosc Percutan Tech 21: 116-119.

38. Lenders JW, Duh QY, Eisenhofer G, et al 2014 Pheochromocytoma and paraganglioma: an endocrine society clinical practice guideline. J Clin Endocrinol Metab 99: 1915-1942.

39. Wang W, Li P, Wang Y, et al, 2015 Effectiveness and safety of laparoscopic adrenalectomy of large pheochro- 
mocytoma: a prospective, nonrandomized, controlled study. Am J Surg 210: 230-235.

40. Kim KY, Kim JH, Hong AR, et al, 2016 Disentangling of malignancy from benign pheochromocytomas/paragangliomas. PloS one 11: e0168413.

41. Rao N, Ramachandran R, Tandon N, Singh P, Kumar R, 2016 Laparoscopic adrenalectomy for pheochromocytoma-does size matter? A single surgeon comparative study. Transl Androl Urol 5: 780-783.

42. Hallfeldt KK, Mussack T, Trupka A, Hohenbleicher F, Schmidbauer S, 2003 Laparoscopic lateral adrenalectomy versus open posterior adrenalectomy for the treatment of benign adrenal tumors. Surg Endosc 17: 264-267.

43. Bozkurt IH, Arslan M, Yonguc T, et al, 2015 Laparoscopic adrenalectomy for large adrenal masses: Is it really more complicated? Kaohsiung J Med Sci 31: 644-648.

44. Rao N, Ramachandran R, Tandon N, Singh P, Kumar R, 2016 Laparoscopic adrenalectomy for pheochromocytoma-does size matter? A single surgeon comparative study. Translational Andrology and Urology 5: 780-783.

45. Zografos GN, Farfaras A, Aggeli C, et al, 2007 Laparoscopic adrenalectomy for large adrenal metastasis from contralateral renal cell carcinoma. JSLS 11: 261-265.

46. Henry JF, Sebag F, Iacobone M, Mirallie E, 2002 Results of laparoscopic adrenalectomy for large and potentially malignant tumors. World J Surg 26: 1043-1047.

47. Zografos GN, Kothonidis K, Ageli C, et al, 2007 Laparoscopic resection of large adrenal ganglioneuroma. JSLS 11: 487-492.

48. Shen WT, Sturgeon C, Clark OH, Duh QY, Kebebew E, 2004 Should pheochromocytoma size influence surgical approach? A comparison of 90 malignant and 60 benign pheochromocytomas. Surgery 136: 1129-1137.

49. Tsuru N, Suzuki K, Ushiyama T, Ozono S, 2005 Laparoscopic adrenalectomy for large adrenal tumors. J Endourol 19: 537-540.

50. Sturgeon C, Kebebew E, 2004 Laparoscopic adrenalectomy for malignancy. Surg Clin North Am 84: 755-774.

51. Pedziwiatr M, Wierdak M, Natkaniec M, et al, 2015
Laparoscopic transperitoneal lateral adrenalectomy for malignant and potentially malignant adrenal tumours. BMC Surg 15: 101.

52. Thompson LD, 2002 Pheochromocytoma of the adrenal gland scaled score (PASS) to separate benign from malignant neoplasms: a clinicopathologic and immunophenotypic study of 100 cases. Am J Surg Pathol 26: 551-566.

53. Conzo G, Musella M, Corcione F, et al, 2012 Laparoscopic adrenalectomy, a safe procedure for pheochromocytoma. A retrospective review of clinical series. Int J Surg 11: 152-156.

54. Fishbein L, Bonner L, Torigian DA, et al, 2012 External beam radiation therapy (EBRT) for patients with malignant pheochromocytoma and non-head and neck paraganglioma: combination with ${ }^{131} \mathrm{I}-\mathrm{MIBG}$. Horm Metab Res 44: 405-410.

55. Edstrom Elder E, Hjelm Skog AL, Hoog A, Hamberger B, 2003 The management of benign and malignant pheochromocytoma and abdominal paraganglioma. Eur J Surg Oncol 29: 278-283.

56. McCauley LR, Nguyen MM, 2008 Laparoscopic radical adrenalectomy for cancer: long-term outcomes. Curr Opin Urol 18: 134-138.

57. Brix D, Allolio B, Fenske W, et al, 2010 Laparoscopic versus open adrenalectomy for adrenocortical carcinoma: surgical and oncologic outcome in 152 patients. Eur Urol 58: 609-615.

58. Lezoche G, Baldarelli M, Cappelletti Trombettoni MM, et al, 2016 Two Decades of Laparoscopic Adrenalectomy: 326 Procedures in a Single-Center Experience. Surg Laparosc Endosc Percutan Tech 26: 128-132.

59. Soulie M, Salomon L, Seguin P, et al, 2001 Multiinstitutional study of complications in 1085 laparoscopic urologic procedures. Urology 58: 899-903.

60. Terachi T, Yoshida O, Matsuda T, et al, 2000 Complications of laparoscopic and retroperitoneoscopic adrenalectomies in 370 cases in Japan: a multi-institutional study. Biomed Pharmacother 54: Suppl 1: 211-214.

61. Zografos GN, Markou A, Ageli C, et al, 2006 Laparoscopic surgery for adrenal tumors. A retrospective analysis. Hormones (Athens) 5: 52-56. 